\title{
Correction to: Regional Metabolic Patterns of Abnormal Postoperative Behavioral Performance in Aged Mice Assessed by ${ }^{1} \mathrm{H}$-NMR Dynamic Mapping Method
}

\author{
Taotao Liu ${ }^{1,2} \cdot{\text { Zhengqian } \mathrm{Li}^{1} \cdot \text { Jindan } \mathrm{He}^{1} \cdot \mathrm{Ning}^{1} \mathrm{Yang}^{1} \cdot \text { Dengyang Han }}^{1} \cdot$ \\ Yue $\mathrm{Li}^{1} \cdot \mathrm{Xuebi}_{\mathrm{Tian}^{3}}{ }^{2}$ Huili Liu ${ }^{2} \cdot$ Anne Manyande $^{4} \cdot$ Hongbing Xiang $^{3}$. \\ Fuqiang $\mathrm{Xu}^{2,5} \cdot$ Jie $\mathrm{Wang}^{2,5,6} \cdot$ Xiangyang Guo $^{1}$
}

Published online: 4 April 2020

(C) Shanghai Institutes for Biological Sciences, CAS 2020

\section{Correction to: \\ Neurosci Bull 2020, 36(1):25-38 \\ https://doi.org/10.1007/s12264-019-00414-4}

The original version of this article unfortunately contained some mistakes.

The authors found that in this article, Figure 10C was a wrong placed figure (it was duplicated with Figure 10D), and the corrected figure was shown as follows (C).

The original article can be found online at https:// doi.org/10.1007/s12264-019-00414-4.

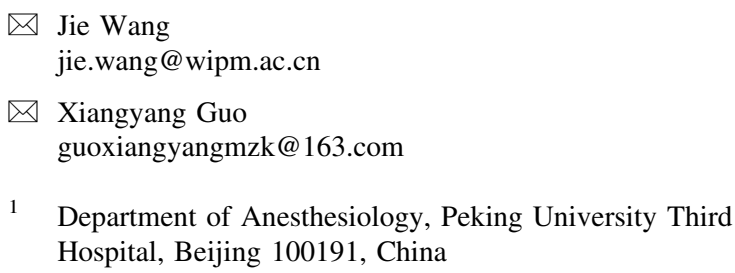

1 Department of Anesthesiology, Peking University Third Hospital, Beijing 100191, China

2 Key Laboratory of Magnetic Resonance in Biological Systems, State Key Laboratory of Magnetic Resonance and Atomic and Molecular Physics, Wuhan Institute of Physics and Mathematics, Chinese Academy of Sciences, Wuhan 430071, China
3 Department of Anesthesiology and Pain Medicine, Tongji Hospital, Tongji Medical College, Huazhong University of Science and Technology, Wuhan 430030, China

4 School of Human and Social Sciences, University of West London, Middlesex TW89GA, UK

5 University of Chinese Academy of Sciences, Beijing 100049, China

6 The Second Hospital of Shijiazhuang, Shijiazhuang 050051, China 

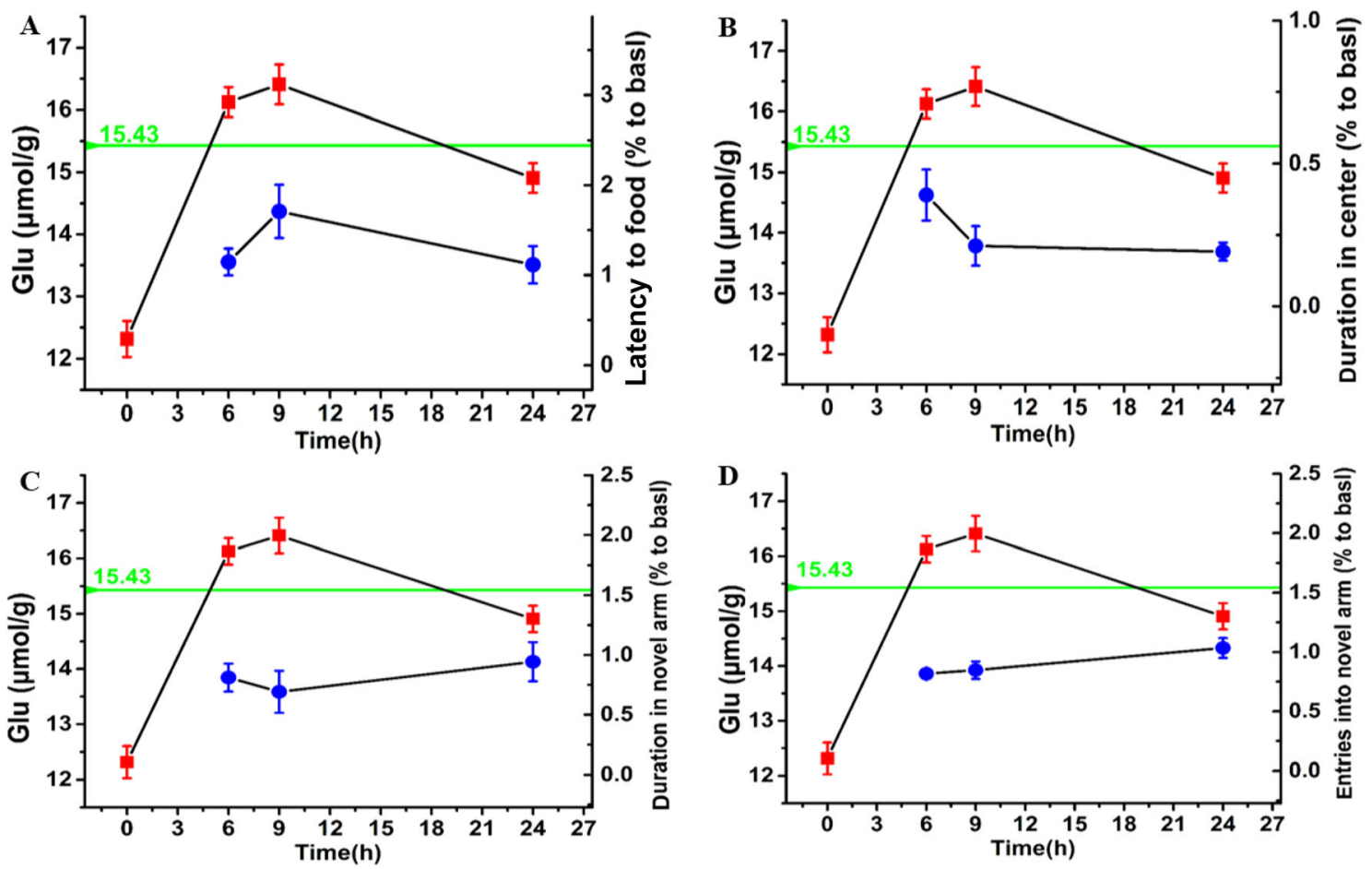